\title{
Deportes electrónicos. Una aproximación a las posibilidades comunicativas de un mercado emergente
}

\author{
Marcos Antón Roncero I marcanto@ucm.es \\ Francisco García García I fghenche@gmail.com \\ UnIVERSIDAD COMPLUTENSE DE MADRID
}

Marcos Antón es licenciado en Comunicación Audiovisual y personal investigador en formación en la Universidad Complutense. Actualmente prepara su tesis doctoral sobre el fenómeno de los deportes electrónicos, un mercado que conoce bien ya que ha trabajado en él como periodista, y ha desempeñado actividades de producción audiovisual en el sector.

Francisco García es catedrático de Comunicación Audiovisual y Publicidad en la Universidad Complutense. Fue director del Centro Nacional de Información y Comunicación Educativa (CNICE), de 4 programas de innovación docente y Presidente de la Asociación de Televisión Educativa Iberoamericana (ATEI). Ha publicado numerosos artículos y dirigido más de 70 tesis.

Resumen: Los deportes electrónicos o esports son el resultado de la profesionalización del mundo competitivo de los videojuegos, un fenómeno reciente que se ha desarrollado con fuerza en los últimos años. Este mercado ha hecho de Internet su pilar fundamental, logrando la exploración y consolidación de nuevas técnicas comunicativas a través de procesos transmedia, la televisión por streaming y el vídeo bajo demanda. En España, así como en otros países europeos y de América del Sur, el sistema de competiciones y patrocinios se está volviendo cada vez más complejo, asemejándose al modelo norteamericano y surcoreano. La proliferación de este sector ha atraído a empresas de diferentes ámbitos que buscan posicionar sus marcas en un mercado emergente que ha demostrado su estabilidad, rentabilidad y potencial de crecimiento.

Palabras clave: Videojuegos competitivos, eSports, Game Studies, Cultura digital, Publicidad

Abstract: Electronic Sports or eSports are the result of the professionalization of the competitive world of video games, a relatively recent phenomenon that has been developed strongly in recent years. This market has made the Internet its cornerstone, making exploration and consolidation of new communication techniques through transmedia procedures, television via streaming and video on demand. In Spain, as well as in other European and South American countries, competitions and sponsorship system becomes increasingly complex, resembling the American and South Korean model. 
The development of this sector has attracted companies from different fields in order to position their brands in an emerging market that has proven to be stable and profitable, while continuing to grow.

Key words: Competitive Gaming, eSports, Game Studies, Digital Culture, Advertising 


\section{Introducción}

La paulatina consolidación de la industria de los videojuegos es un hecho avalado por las cifras de facturación de este mercado (Martí, 2010: 11). Según los datos publicados por la Asociación española de Distribuidores y Editores de Software de Entretenimiento (aDeSe), el mercado del videojuego español es el cuarto con mayor consumo de Europa, con una facturación de 822 millones de euros, (Adese, 2013: 7); una cifra que supera los 614 millones del cinematográfico o los 172 de las artes escénicas (MCU, 2013: 311). La vertiente competitiva dentro del mundo de los videojuegos cobra cada vez más importancia y el nivel de profesionalización en el ámbito de los deportes electrónicos o eSports va en aumento, lo que ha llevado a muchas empresas a buscar el posicionamiento de su marca en un mercado en pleno desarrollo en España y América del Sur, pero ya consolidado en algunos países europeos, Estados Unidos y Corea del Sur.

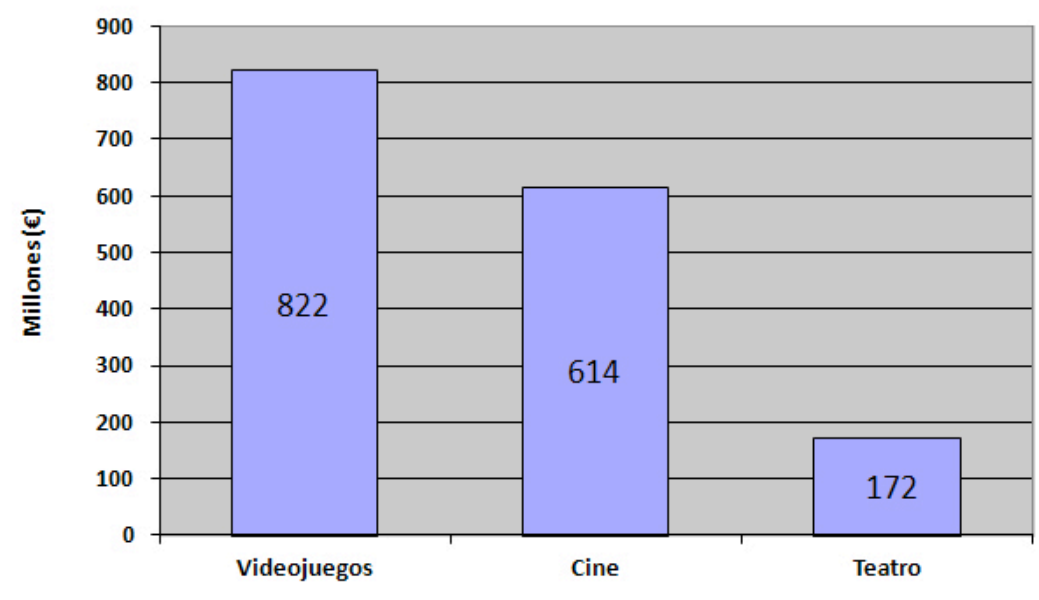

Tabla 1. Comparativa en las cifras de facturación

En un panorama protagonizado por la convergencia multimedia, los anunciantes buscan abrirse paso en nuevos mercados para llegar a los nuevos públicos. En los últimos años se ha registrado un incremento de la participación de empresas ajenas a la informática y las Tecnologías de la Información y la Comunicación (TIC) en el nicho publicitario de los deportes virtuales, sumándose así a las corporaciones tecnológicas ya presentes. El fenómeno se encuadra dentro de un sector ya de por sí innovador que experimenta con alternativas desde el punto de vista comunicativo, explorando segundas pantallas y generando un consumo televisivo a través de nuevas vías como el streaming ${ }^{1}$ y el vídeo bajo demanda. Los objetivos de los productores de videojuegos son muy similares a los de cualquier otra empresa: vender un producto y fidelizar al consumidor. Con la inclusión del

\footnotetext{
${ }^{1}$ La técnica de streaming hace referencia a la distribución de contenido multimedia a través de Internet de forma que un usuario puede consumir un producto al mismo tiempo que se lo descarga en su dispositivo como flujo de datos de forma temporal. (MEC, 2008).
} 
espectáculo que rodea a los grandes deportes de masas y el público eminentemente juvenil de los esports, tenemos un resultado muy seductor para las organizaciones que buscan un público objetivo determinado.

\section{Objetivos y metodología}

El presente artículo propone una aproximación al mercado de los deportes electrónicos y plantea una reflexión acerca de las oportunidades que se abren en las áreas de conocimiento de la Comunicación Audiovisual y la Publicidad en este ámbito. Si bien hay autores que han abordado este fenómeno (Marcano, 2012; Auz, 2012; Wagner, 2006), la falta de producción científica en el estudio del fenómeno hace necesaria la existencia de investigaciones que traten de aclarar conceptos para facilitar el examen de este hito de la cultura popular. El artículo pretende analizar las características básicas del mercado y sentar un marco común de referencia que sirva para su comprensión y facilite posteriores investigaciones sobre los diferentes aspectos implicados. Para ello, el análisis se presenta en dos fases transversales: la primera introduce de manera sintética la evolución y recorrido de los deportes virtuales y su alcance e implicación desde un punto de vista comunicativo y social, con el objetivo de servir de marco contextual para la comprensión del ámbito estudiado. La segunda aproximación se realiza en base al examen de diferentes casos concretos que permiten observar la popularización y espectacularización de los deportes electrónicos, estudiando las consecuencias y el desarrollo en la producción audiovisual de este tipo de eventos y la progresiva ampliación del mercado publicitario.

Se atenderá a la descripción y explicación de este fenómeno contemporáneo en el que el investigador estudia el medio como observador no participante de la situación en su contexto natural, lo que permite un análisis cualitativo y una indagación con múltiples fuentes de evidencia que lo facilitan. Es necesario un acercamiento conceptual previo para la comprensión de este mercado y de la adaptación a otros países, especialmente España, de un modelo de explotación consolidado en Norteamérica y Corea. De la misma manera, se estudian las características comunes que convierten a un videojuego en un deporte electrónico y se hace un repaso de los principales hitos publicitarios de las marcas que participan en este mercado.

\section{Posibilidades comunicativas y comerciales de un mercado emergente: los eSports}

El nacimiento y evolución del mercado de los videojuegos comienza en la década de los ochenta, de forma paralela a la consolidación de la informática de consumo en los hogares y la informatización de los entornos de trabajo. "Desde entonces, la industria del videojuego se ha ido convirtiendo en un próspero negocio y en un importante elemento de la cultura popular" (Gómez, 2007: 72). 
La interactividad y competitividad entre jugadores de videojuegos ya había quedado patente durante los años setenta. Muestra de ello es el primer torneo oficial celebrado en la Universidad de Standford en 1972, en torno al juego Spacewar (Brand, 1972). Es en junio de 1997 cuando surge en Estados Unidos la que está considerada como la primera agrupación deportiva en celebrar campeonatos profesionales, la Cyberathlete Professional League (CPL), encargada de organizar ese mismo año su primer torneo con un modesto premio de 4.000 dólares (CPL, 2014). En menos de dos décadas, el alcance y la repercusión de las competiciones han sufrido un crecimiento acelerado. Muestra de ello fue la final mundial de la tercera temporada de la League of Legends Championship Series (LCS) ${ }^{2}$, celebrada en el prestigioso estadio Staples Center de Los Ángeles. En este campeonato, el equipo de veinteañeros surcoreanos SK Telecom T1 se hizo con el premio de un millón de dólares (Lang, 2013) en una emisión que registró más de 30 millones de espectadores (Trasgo, 2014).

La llegada de Internet a los hogares revolucionó el sector y pronto añadió un aliciente al juego: la posibilidad de competir contra otras personas en línea gracias a los modos multijugador, lo que llevó a la creación de grandes comunidades de jugadores organizadas en torno a un título cuyos objetivos eran compartir "las experiencias de juego (...) invitar a partidas entre equipos y mantenerse informados de las noticias importantes" (Marcano, 2012: 113).

La popularidad que alcanzaron las competiciones en Corea del Sur, llevó a la creación de la Korean eSports Association (KeSPA) en el año 2000. KeSPA surgió como una asociación de representantes del panorama surcoreano de los deportes electrónicos que avalaba de forma oficial a los jugadores profesionales en un país donde algunos de estos torneos se retransmiten a través de la televisión pública (Kalning, 2007; Red Apple Entertainment, 2006). La situación de Corea era, hasta hace poco, un hecho aislado en el panorama internacional; en este país, algunos jugadores de videojuegos como StarCraft $^{3}$ son verdaderas estrellas mediáticas, protagonistas de spots televisivos y poseedores de una amplia comunidad de fans similar a la de otros deportes tradicionales en países occidentales (Red Apple Entertainment, 2006). Pronto el fenómeno se extendió por Norteamérica y países como Suecia y Alemania. Por entonces, los estudios sobre videojuegos aún subrayaban sus aspectos negativos, como ya ocurriera en su día con el cine, la radio o la televisión (Montero y Rueda, 2001: 79).

\footnotetext{
${ }^{2}$ League of Legends es un videojuego gratuito producido, por Riot Games. Encuadrado dentro del género MOBA (Multiplayer Online Battle Arena) con influencias de RTS (Real Time Strategy) y los juegos de rol o RPG (Role-Playing Game), consiste en el enfrentamiento entre dos equipos de cinco jugadores. Cada jugador controla un personaje que ha de combinar sus habilidades con las del resto de jugadores del equipo para lograr la conquista de la base contraria.

3 StarCraft es un videojuego RTS (Real Time Strategy) de ciencia ficción desarrollado por Blizzard Entertainment, empresa creadora de otros títulos como World of Warcraft o Diablo, que consiste en la gestión de los recursos y unidades de la colonia de un jugador para conseguir la victoria sobre el oponente.
} 
Estados Unidos ha desarrollado, de manera paralela a Corea, uno de los principales mercados dedicados a los eSports. Actualmente, Major League Gaming (MLG) es la compañía responsable de la que está considerada la liga más importante del mundo: la MLG Pro Circuit; de MLG.tv, la primera cadena de televisión online dedicada al videojuego competitivo profesional, y de MLG: Play, la mayor plataforma de torneos online, con 9 millones de jugadores registrados (MLG, 2013). En España y América del Sur, el terreno para el desembarco de los deportes digitales fue preparado por grandes festivales digitales conocidos como LAN Party que incluían en su programación torneos de videojuegos. El auge y difusión de estas macro-concentraciones en las que aficionados a la informática se reúnen para intercambiar información y jugar a videojuegos, ha alcanzado gran popularidad en los últimos años (Ramírez, 2008: 17). Son ejemplo de ello algunas citas como Campus Party en Valencia, México o Ecuador, así como otras de alcance nacional como Euskal Party (actualmente Euskal Encounter), o Tenerife LAN Party.

En el panorama español conviven diferentes agentes del sector de los deportes digitales entre clubes, responsables de eventos competitivos y otro tipo de compañías como productoras audiovisuales. Algunas de las ligas con mayor popularidad son las organizadas por Liga de Videojuegos Profesional (LVP), Electronic Sports League Spain (ESL) y DreamHack Spain (DH). La primera de ellas fue creada en España en 2009, forma parte de la Federación Internacional de Deportes Electrónicos (IeSF) y es la más joven de las tres; ESL Spain es la división española de una multinacional con más de una década de historia, radicada en Alemania y con presencia en más de 60 países (Trasgo, 2014), y DH es la versión nacional de una organización sueca formada en 1994 y responsable de la que actualmente está considerada la mayor LAN Party del mundo (Pascual, 2013). Durante los últimos años han proliferado otras ligas organizadoras de competiciones de videojuegos a iniciativa de organizaciones como Euro Gamers Online, Tenerife Lan Party o SocialNat, que han pasado a dinamizar el sector, así como torneos menores de comunidades de jugadores como Arcadia Fighters o SupplyZero, además de otras competiciones celebradas en diferentes ámbitos, como el universitario. Según Sebastian Radu, CEO de ESL Spain, "la entrada en escena de los nativos digitales" ha sido vital para el desarrollo del fenómeno en España (Trasgo, 2014).

Estas organizaciones proporcionan el soporte tecnológico necesario para que los jugadores puedan competir en una plataforma común y se financian de diferentes maneras: además del capital propio y los ingresos por publicidad y patrocinadores, el modelo más extendido es el pago por inscripción, en el que el jugador o equipo paga una cuota para poder participar y optar a los premios. También funciona el modelo de apuesta en el que "cada jugador apuesta una cantidad y el ganador se lleva el 90\%, mientras que la página organizadora se queda con el 10\% restante" (Del Palacio, 2012). 


\subsection{Espectacularización del fenómeno}

Durante los últimos diez años, los deportes electrónicos han crecido de forma exponencial, tanto cuantitativa como cualitativamente, llegando a la espectacularización del fenómeno. Así lo expresaba, durante una entrevista, Sergi Mesonero, director de la Liga de Videojuegos Profesional (LVP):

Nos hemos beneficiado de un cierto cambio de perspectiva en lo que respecta a los deportes digitales (...). En los últimos dos o tres años, y gracias a títulos como League of Legends o Call of Duty ${ }^{4}$, los deportes digitales se han extendido a una masa muchísimo más importante de gente. Entienden qué es el concepto de eSport y se han aficionado, no solo a competir sino lo que es más importante: a convertirse en espectadores y a disfrutar del espectáculo. (entrevistado en Fernández, 2013).

Los datos demuestran por sí solos la popularidad actual de los deportes electrónicos. Solo League of Legends alcanzó en 2012 los 32 millones de jugadores mensuales, una cifra que le avala como el videojuego con mayor número de jugadores del mundo; una cifra solo 8 millones inferior al total de suscriptores mensuales de Xbox (Riot, 2012).

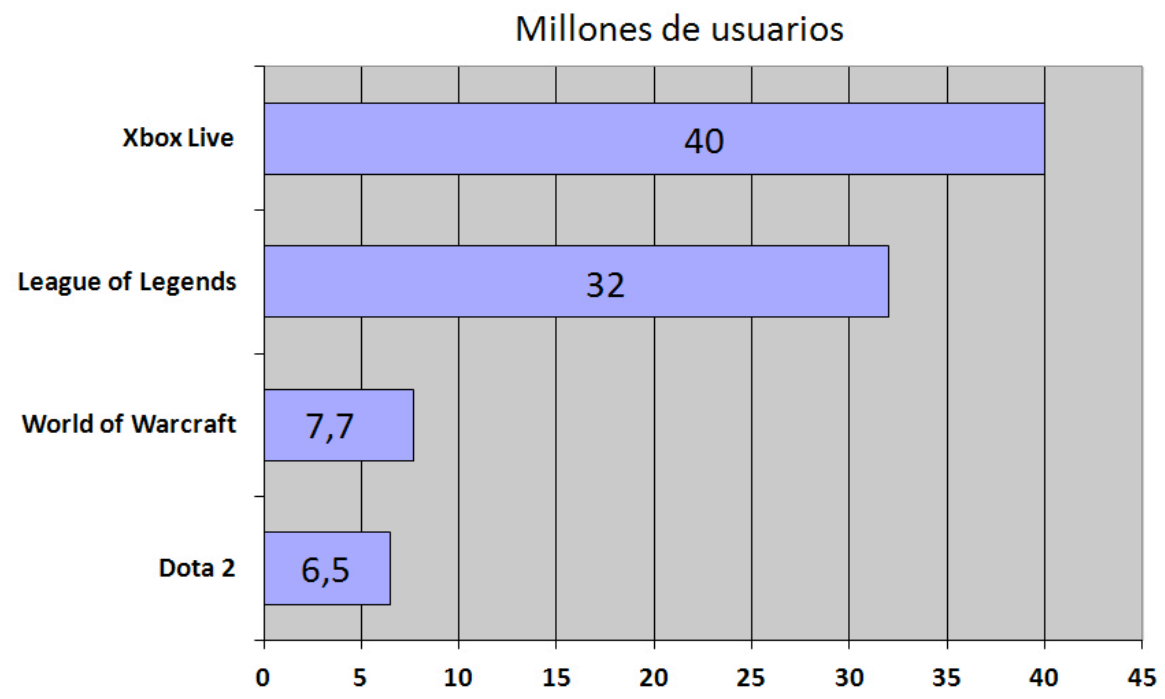

Tabla 2. Millones de usuarios mensuales activos.

Millones de jugadores amateur aspiran a convertirse en profesionales, una tarea que no es sencilla y que propicia iniciativas como la llevada a cabo por los artífices de DH España, quienes han lanzado un producto Ilamado Academia eStar, una academia para formar jugadores profesionales (Carabantes, 2014).

No existe una clasificación consensuada sobre qué juegos pueden ser considerados eSports, pero si hay un acuerdo tácito entre los diferentes agentes que participan de este fenómeno, así como intentos de algunos medios para acotar las características que ha de tener un

4 Call of Duty es una saga de videojuegos de disparos en primera persona (first person shooter) distribuida por Activision. 
videojuego para ser considerado como un deporte digital (Lang, 2013; Fernández, 2013; Kalning, 2007). En base a la observación de las competiciones existentes, podemos establecer unas características comunes que convierten a un videojuego en eSport:

- Ha de ser popular y gozar de la participación activa de una masa de espectadores, generalmente agrupados en torno a una comunidad, quienes además de ser jugadores, son productores y consumidores de información relativa al juego.

- Permite el enfrentamiento en línea entre dos o más jugadores. Batirse contra la máquina no se considera un enfrentamiento competitivo. Las partidas se realizan a través de un servidor y las condiciones de victoria son establecidas en base a las reglas propias del juego y a la normativa establecida por los responsables deportivos de la competición, una normativa que puede ser propia o adaptada de otros reglamentos como el de MLG o el de Decerto. Hay árbitros que hacen cumplir la aplicación del reglamento y sancionan las infracciones.

- Existen tres niveles de competición: amateur, semiprofesional y profesional. Jugar habitualmente a videojuegos no es sinónimo de ser jugador profesional. La motivación de un pro-gamer no es únicamente la diversión sino que sus valores son similares a los de un deportista tradicional. "Exhiben la unidad competitiva de un atleta, la habilidad táctica de un general y el talento creativo de un artista" (Nine, 2013). Los jugadores entrenan diariamente, desarrollan sus habilidades y estudian a sus rivales, unas técnicas presentes en otros deportes (Carabantes, 2014; Del Palacio, 2012). "Entrenar no significa jugar mucho, significa jugar de una manera estructurada" (Mesonero entrevistado en Fernández, 2013).

- Los jugadores suelen pertenecer a una organización y pueden estar agrupados en diferentes niveles; de menor a mayor dimensión: plantillas, equipos y clubes.

- Los jugadores con un nivel de profesionalización más alto tienen una completa dedicación hacia el videojuego, llegando a poder vivir de ello a través de un salario, de los premios y/o de los ingresos obtenidos de sus patrocinadores (Carabantes, 2014). "Este es su trabajo, su forma de hacer dinero; trabajan tan duro como los atletas profesionales" (Nine, 2013).

- Existe un mercado real de jugadores, entrenadores, equipo técnico, comercial, etc., con movimientos, fichajes y contratos. Los jugadores más cotizados suelen desempeñar su labor en países con ligas más desarrolladas como Corea o Estados Unidos. Los mejores jugadores europeos suelen agruparse en conjuntos comunitarios para participar en ligas internacionales, como ocurre con el conjunto Fnatic, donde milita el español Enrique Cedeño (xPeke) o Millenium, ex equipo de Alvar Martín (Araneae). 
- Las competiciones ofrecen premios que varían en función de su prestigio y sus patrocinadores, desde el millón de dólares para el vencedor de la LCS (Lang, 2013), hasta premios más modestos de las competiciones españolas celebradas a finales de 2013, como los 2.750 euros en la Final Cup 5 de LVP para el vencedor de League of Legends o los 1.400 euros de la Serie A de Call of Duty organizada por Euro Gamers Online.

- Por lo general, desarrolladores y responsables de un juego muestran cierta predisposición para establecer su producto como un eSport en el mercado a través de la cesión de licencias, patrocinios de torneos y publicidad.

La conjunción de estas características confiere a un videojuego el estatus de deporte electrónico, y son la causa de que unos títulos dominen por encima de otros en las competiciones. Hay ciertos géneros que incitan a la competición como son los simuladores deportivos (FIFA), los shooters o videojuegos de disparos (Call of Duty, Counter Strike, Halo, ShootMania o World of Tanks) y los juegos de estrategia y rol (StarCraft, League of Legends, Defense of the Ancients, World of Warcraft, o Age of Empires). La mayoría tienen alcance global, pero los gustos difieren en función de los países. Sucede, por ejemplo, con juegos como StarCraft II, el más popular en las ligas coreanas (Wagner, 2006: 437), o Halo 4, hasta hace poco el shooter por antonomasia en Estados Unidos, y que ha quedado relegado de competiciones como la MLG por Call of Duty, el favorito en España. Los expertos del sector señalan los cambios cíclicos en la popularidad de los juegos. Así lo explica Mesonero:

Siempre tiene que existir el juego de referencia. Hace diez años serían StarCraft I y Counter Strike, hace cinco hubo un crecimiento brutal de World of Warcraft competitivo, y ahora le toca a Call of Duty y League of Legends. Posiblemente dentro de otros cinco años serán otros títulos diferentes" (entrevistado en Fernández, 2013).

3.2. Hacia un nuevo paradigma de televisión: vídeo bajo demanda, streaming y producciones profesionales

En el panorama actual de convergencia multimedia en el que nos encontramos, "el modo en el que la televisión se ve ha cambiado. (...) Los dispositivos móviles han puesto en bandeja, a través del desarrollo de las telecomunicaciones e Internet, la posibilidad de acceder tanto a las emisiones de televisión como a contenidos y productos adicionales que complementan la experiencia televisiva" (Barrientos, 2013: 379). Paralelamente al desarrollo de los eSports se ha gestado un mercado televisivo alternativo que se ha servido de los avances tecnológicos multiplicando el alcance de ligas y torneos, ya que los medios generalistas han sido reacios a la cobertura de estos eventos, como explica Mesonero: "Con el tema de los videojuegos hay un poco de miedo, hay un poco de «no veo a nadie con katana»". Los medios tradicionales, "no entienden muy bien de qué se trata, y cuando vienen no saben a qué atenerse" (entrevistado en Fernández, 2013). Esta situación ha llevado a los organizadores de ligas y torneos a formar sus propios equipos de producción audiovisual para suplir la demanda de la 
comunidad. Aprovechándose del fenómeno transmedia, han establecido sus propios canales de televisión a través de técnicas como el vídeo bajo demanda y la retransmisión por streaming. "Hace tres años, los streamings eran imposibles o muy difíciles", afirma Mesonero, "gracias a las mejoras en infraestructura tecnológica, el espectáculo de los eSports se ha podido llevar a todas las casas " (entrevistado en Fernández, 2013). Este modelo lleva años de desarrollo en los mercados surcoreano y norteamericano. Según los datos de MLG.tv, su audiencia ha crecido un $600 \%$ en los últimos 3 años, y el consumo de vídeo online un $1557 \%$ desde 2011 . Un consumo que los usuarios realizan a través de Internet, desde dispositivos móviles como teléfonos y tabletas, consolas o televisores conectados a la Red (MLG, 2013).

Al mismo tiempo, ha crecido el número de prosumidores, fans que se han convertido en productores de contenidos ante la falta de oferta; una tendencia aprovechada por plataformas dedicadas a la retransmisión de vídeo bajo demanda, siguiendo el modelo de YouTube. Estas plataformas, conocidas como live streaming video platforms, han sabido adaptarse a este tipo de usuarios para desarrollar su negocio. Es el caso de Twitch.tv, la plataforma especializada en videojuegos y eSports creada en 2011 por Justin.tv que se ha establecido como líder del sector, con 45 millones de visitantes mensuales y una media de 100 minutos de vídeo al día. Con tan solo dos años de vida y un centenar de empleados, Twitch adelanta a servicios más veteranos como Hulu, una plataforma de contenidos audiovisuales respaldada por Disney, NBC Universal y 21st Century Fox, que cuenta con 30 millones de visitantes mensuales (Ewalt, 2013).

Hoy en día, Twitch.tv ofrece desde retransmisiones caseras de las partidas de sus usuarios hasta grandes campeonatos internacionales. La revista Forbes recoge en su artículo las declaraciones de Emmett Shear, cofundador de Justin.tv y CEO de Twitch.tv: "Nos dimos cuenta de que no éramos buenos creando contenido y dejamos que la gente lo hiciera. Nunca hubiéramos imaginado en lo que se convirtió" (Ewalt, 2013). Twitch.tv es actualmente el referente de las retransmisiones de eSports, un modelo que YouTube también ha adaptado durante 2013. Lo mismo han hecho la mayoría de ligas, quienes ofrecen a través de sus portales auténticas producciones televisivas, llegando a establecer grandes set ups profesionales de emisión, como el de MLG (Nine, 2013). Los deportes virtuales han apostado por este innovador modelo transmedia como alternativa a la televisión tradicional para llegar a los nativos digitales. "Hay quienes nos preguntamos si dentro de diez años tendrá sentido la televisión tal cual la conocemos", afirma Mesonero (entrevistado en Fernández, 2013). La interactividad conseguida gracias al chat en tiempo real entre los usuarios y de estos con sus ídolos es una de las claves del éxito. Según declaraciones del analista de Wedbush Securities, Michael Patcher, "Twitch dio una solución para quienes demandaban contenidos en tiempo real, permitiéndoles ser espectadores e interactuar al mismo tiempo. Nadie había hecho eso antes" (Ewalt, 2013). 
En España y América del Sur, ha crecido la oferta de canales de streaming y vídeo bajo demanda gracias a la ventaja de poseer una lengua común, el español, que permite romper las barreras geográficas. Los datos demuestran que el crecimiento de expectación de los eventos de esports ha aumentado un $20 \%$ en los dos últimos años; es el caso de LVP cuyo primer evento congregó a menos de 400 personas mientras que en la Final Cup 5 celebrada en octubre de 2013 se vendieron 8.000 entradas (Carabantes, 2014). Pero sin duda, la verdadera oportunidad está en la fuerza del streaming: la retransmisión de la Final Cup 4, en mayo del mismo año, alcanzó la cifra de 100.000 espectadores únicos (Fernández, 2013).

\subsection{Oportunidades comunicativas: periodismo deportivo ciudadano.}

Ya se ha hecho referencia en este trabajo a la reticencia por parte de los medios de comunicación generalistas a entrar en el ámbito de los eSports (Fernández, 2013). Esta es una de las causas que ha contribuido al desarrollo de la figura del prosumidor mediático dentro de las comunidades de jugadores y fans. Han surgido numerosas plataformas de información en páginas web, foros y redes sociales que albergan contenidos de muy diversa índole, desde noticias sobre competiciones y fichajes, pasando por agendas de actividades, hasta análisis, comentarios de partidas o reportajes, libros y documentales sobre el mundo de los deportes digitales. El establecimiento progresivo de estos medios de comunicación alternativos en España ha generado interés y multiplicado la demanda informativa, lo que ha llevado a la creación de nuevos espacios informativos disgregados sin grandes líderes ni medios de comunicación dominantes, como sí ocurre en Estados Unidos (Ewalt, 2013). Las fuentes emisoras encargadas de la producción de esta información se pueden clasificar en diferentes grupos comunicativos:

- Organizaciones responsables de competiciones y torneos. Todas las organizaciones (LVP, ESL, DH...) poseen su plataforma informativa.

- Información institucional y/o personal de jugadores y equipos profesionales. La gran mayoría de clubes (Karont3, Giants, Dimegio...) tienen su propia plataforma web a través de la cual informan a su comunidad y desarrollan su imagen de marca. Lo mismo ocurre con los jugadores más populares quienes mantienen una continua actividad en las redes sociales como ocurre con Pedro Moreno (LucifroN) o Carlos Rodríguez (Ocelote).

- Comunidades organizadas, jugadores no profesionales y fans. Ya hemos citado ejemplos de comunidades como Supplyzero o Arcadia Fighters, pero también entraría en esta categoría los denominados Youtubers, usuarios populares de la plataforma de Google que también realizan contenidos relacionados con los eSports.

- Publicaciones especializadas con vocación periodística y propósitos profesionales. Pueden ser de reciente creación o bien fruto de la apertura de un nuevo nicho de 
negocio por parte de empresas del sector. Estas dos vertientes han proliferado en los últimos años y hay varios ejemplos como el periódico digital Trasgo.net, el grupo argentino Rage Interactive Group, o la productora UnTTV Prods., quien además de proveedora de servicios para competiciones como la Final Cup o la ESL Pro Series, produjo uno de los primeros informativos de esports en habla hispana.

- Publicaciones especializadas de videojuegos que deciden introducirse en el mundo de los deportes electrónicos. El caso paradigmático en España es MeriStation, perteneciente al grupo Prisa, o IGN España, de Unidad Editorial, pero existen otros ejemplos como Hobby Consolas o 3DJuegos.

- Medios generalistas. Ha habido diferentes acercamientos a este fenómeno por parte de medios consolidados (cobertura de eventos por parte de Antena 3, El Mundo o cadenas regionales y locales). Especialmente significativo es el caso de Canal+, quien a través de Canal+ Xtra y de Yomvi, su servicio a la carta, han ofrecido las últimas Final Cup de LVP, o el reciente acuerdo entre esta Liga y el Grupo Mediaset, para la emisión de los partidos a través de Mitele.es.

- Agentes externos o empresas de otros sectores que invierten en el mundo de los deportes digitales y se convierten en productores de información. Es el caso de compañías como Movistar, Vodafone, Red Bull, Coca Cola, American Express, FNAC o El Corte Inglés.

3.4. Oportunidades publicitarias: el posicionamiento de la marca en un mercado emergente.

El sector de los videojuegos en general y de los deportes electrónicos en particular presenta, para los anunciantes, las oportunidades de un mercado emergente en el cual el posicionamiento de una marca es vital en los primeros estadios. Diferentes estrategias y técnicas publicitarias tienen cabida en un sector basado en el desarrollo tecnológico e Internet. Muchas marcas desarrollan estrategias de comunicación pull, buscando que el consumidor acuda al contenido publicitario (Martorell y Rom, 2011: 25); por ello, en los eSports son especialmente utilizadas las estrategias publicitarias en la Red combinadas con las usadas en los eventos deportivos y el sector de los videojuegos ${ }^{5}$. Para los expertos, el crecimiento de los deportes electrónicos será continuo gracias a los nativos digitales, quienes harán de ellos un nicho de mercado estable y bastante considerable (Carabantes, 2014; Trasgo, 2014). "Es difícil que las personas mayores de 50 años se aficionen (...), pero el público veinteañero actual seguirán siéndolo en el futuro, y quienes ahora tienen diez años pronto se convertirán en espectadores" (Ewalt, 2013). No obstante, hay investigaciones que subrayan la popularización que los videojuegos han logrado en grupos no asociados tradicionalmente con esta actividad (Gómez y Navarro, 2013: 33).

\footnotetext{
${ }^{5}$ Puede ampliar información sobre estas estrategias en Martí (2010).
} 
Esta idea no parece equivocada teniendo en cuenta los últimos movimientos en el patrocino de competiciones y equipos. Las compañías relacionadas con el mundo de los videojuegos (Sony, Microsoft, Blizzard, Riot) o las grandes multinacionales tecnológicas (Intel Corporation, Olympus, Hewlett-Packard) ya no son los únicos patrocinadores de las competiciones. Pequeñas y medianas empresas del sector como MadCatz, PC Box, RedCoon (patrocinadores de la LVP) o Versus Gamers, tienda online de hardware, forman parte del entramado comercial. También están presentes empresas de telecomunicaciones como Telefónica, patrocinadora de diferentes eventos desde hace varios años (su edificio de la Gran Vía de Madrid albergó las finales de la décima temporada de la ESL Pro Series), o Vodafone y ONO, patrocinadores de la LVP. De la misma manera, algunas administraciones públicas colaboran en el sector como es el caso de DreamHack y el Ayuntamiento de Valencia, o el Consistorio de Barcelona con LVP, cuyas Final Cup 4 y 5 fueron celebradas en el Disseny Hub.

El incremento de la popularidad y la espetacularización de las competiciones han despertado el interés de otras empresas ajenas al sector. Compañías como FNAC o El Corte Inglés han acogido en sus edificios actividades relacionadas directa o indirectamente con la LVP en Barcelona o a las ESL Pro Series en Madrid. En las últimas competiciones celebradas en España se ha visto la incursión de marcas como Filipinos, producto de Galletas Artiach, que en 2013 ofreció patrocinio al equipo más popular de la cuarta temporada de la LVP; los usuarios podían votar a través de los códigos impresos en el envoltorio del producto. Siguiendo esta tendencia, la empresa Risi publicitó su aperitivo Risketos en la retransmisión de las primeras finales de la Serie A de Euro Gamers Online.

En Estados Unidos, Intel o American Express son patrocinadores oficiales de algunos torneos emitidos a través de Twitch, y medios de comunicación como Wired, IGN o Joystiq mantienen canales en la plataforma, creando contenido original y consiguiendo un aumento de la audiencia (Ewalt, 2013). Mención especial merece el caso de la marca Red Bull, conocida por su constante participación en el patrocinio de deportes de motor y aventura. La compañía ha entrado con fuerza en el panorama internacional de los deportes electrónicos en los últimos años. Actualmente, su página web alberga una sección dedicada a los deportes digitales, y en noviembre de 2013 organizó el torneo Red Bull Battle Grounds New York City. También en el cartel de la Final Cup 4 se podía leer la leyenda "Red Bull Final Cup", un hecho significativo si tenemos en cuenta que la tercera edición había sido patrocinada por uno de sus competidores: Monster Energy Drink. Por su parte, Coca Cola lanzó el patrocinio de la liga Coca Cola Zero Challenger Series 2014 con el objetivo de alcanzar el target de los fans de League of Legends, mayoritariamente compuesto por hombres de 17 a 30 años, tal y como anunció la compañía en la Digitial-Life-Design Conference Münich 2014.

Por otro lado, podemos observar un claro trabajo de marca por parte de los clubes. Es el caso de Karont3 o Giants, equipos españoles que no solo buscan los mejores jugadores "sino 
también aquellos que proporcionen una imagen, pues importa la marca", o de jugadores como Carlos Rodríguez, quien "ha decidido crear lo que considera una imagen de marca" con su apodo, Ocelote. También juegan un papel importante los llamados partners, empresas que insertan publicidad en los vídeos que los jugadores publican en Internet. Es el caso de Alejandro Marcos (SdOw), campeón nacional de Call of Duty que explota su imagen a través de la Red; su partner, la compañía estadounidense Machinima, le paga una cantidad por cada 1.000 visitas recibidas en su canal de YouTube (Del Palacio, 2013).

Cada vez son más las compañías que diseñan ligas y torneos dirigidos a un target determinado. La norteamericana MLG es, a día de hoy, referente mundial. Su canal se promociona como la vía ideal para los anunciantes que deseen llegar a un público compuesto por un $90 \%$ de hombres, un $50 \%$ de jóvenes entre 16 y 34 años, y donde el $76 \%$ son mayores de 21 años. Esta muestra representó entre 2011 y 2012 un total de 15 millones de espectadores únicos procedentes de 170 países diferentes y con un promedio de 150 minutos cada uno (MLG, 2013). Una estrategia para captar anunciantes similar a la de las cadenas de televisión tradicionales, pero que adapta las características del concepto transmedia.

Según el estudio InfoAdex, la inversión publicitaria en videojuegos en 2012 fue de 26,2 millones de euros. Dentro de ese porcentaje, la inversión en Internet registró un aumento del 54\% respecto a 2011 (Adese, 2013: 22). Estas cifras demuestran la potencialidad de una industria, la de los videojuegos, de la que los esports forman parte, y de un medio, Internet, que es su principal pilar. "Las marcas se están dando cuenta de que tienen en los deportes electrónicos un target de gente que es poco accesible por los medios tradicionales; son nativos digitales y su medio de información es Internet" (Carabantes, 2014).

\section{Conclusiones y discusión: un fenómeno por explotar}

El proceso de convergencia mediática y la democratización informativa han revolucionado el sector de los videojuegos elevando a los deportes electrónicos a un nivel nunca antes visto, no solo en cuanto a la popularidad mediática sino también a la rentabilidad económica y a su impacto publicitario.

Además, la concepción académica hacia los videojuegos está cambiando y empieza a observarse el análisis comunicativo de los mismos, a la vez que se reinventan los enfoques desde la ludología, la sociología o la psicología. La vertiente competitiva ha desarrollado un complejo proceso de profesionalización que está contribuyendo a un cambio en la percepción que la sociedad tiene de ellos. La llegada de los nativos digitales a este mercado ha supuesto la eclosión de un fenómeno que llevaba gestándose varias décadas y que ofrece grandes posibilidades, tanto comunicativas como comerciales, gracias a la creciente demanda. 
Por último, cabe señalar que en diferentes países de Asia, América del Sur y Europa, los esports aún se encuentran en pleno desarrollo, pero están avalados por el éxito en mercados consolidados, como el surcoreano y norteamericano, y por algunas experiencias positivas en Alemania y los países escandinavos. Por lo tanto, los deportes digitales se plantean como un mercado en potencia con grandes posibilidades durante los próximos años. En esta línea, se echa en falta una apuesta firme por parte de los grandes medios de comunicación para explorar la potencialidad de este fenómeno, más allá de las tímidas y esporádicas aproximaciones.

Dada la falta de producción científica al respecto y la necesidad de investigar el fenómeno, este artículo plantea diferentes líneas de trabajo futuras:

- Un análisis exhaustivo de las causas sociales y comerciales que han llevado a la popularización de este mercado, la generación del fenómeno fan asociado a él y sus posibilidades comunicativas y de explotación publicitaria.

- Profundización en el estudio de la convergencia comunicativa y la narrativa transmedia en la generación de fenómenos comunicativos audiovisuales alternativos gracias al uso de Internet y a técnicas como el streaming o el vídeo bajo demanda, que conducen hacia los nuevos modelos de televisión.

- Estudios enmarcados en otras disciplinas como la educación, sociología o la psicología que analicen la influencia y las posibilidades potenciales de los deportes electrónicos en los procesos de socialización de los nativos digitales.

\section{REFERENCIAS BIBLIOGRÁFICAS}

ADESE (2013): "BALANCE ECONÓmico de la industria del Videojuego 2012", 12 de MARzo DE 2013, EN HTTP://BOLETINES.PRISADIGITAL.COM/PRESENTBALANCE.PDF (CONSULTADO: 29/01/2014).

Auz, Luis Fernando (2009): "Plan de negocios Para la CREAción de una ARENA de JUegos en red (lan House)", Quito, Escuela Politécnica nacional. Disponible en HTTP://BIBDIGITAL.EPN.EDU.EC/HANDLE/15000/1440 (CONSULTADO: 15/01/2014)

BarRientos, Mónica (2013): "LA SEgunda PANTALLA teleVisiva: La APLiCACión ANT 3.0 DE ANTENA 3", EN ICONO 14, VOL. 11 (2), NO 2, 2013, PP. 357-383. ISSN: 16978293. DOI: RI14.V11I2.568.

Brand, Stewart (1972): "Spacewar: Fanatic life and Symbolic Death Among the Computer Bums", en Rolling Stone, 7 de diciembre de 1972, Disponible en HTTP://WWW.WHEELS.ORG/SPACEWAR/STONE/ROLLING_STONE.HTML (CONSULTADO: 06/01/2014). 
Carabantes, Andrés (2014): "eSports en España" en Cadena Ser, 16 de enero de 2014. DISPONIBLE EN: HTTP://WWW.CADENASER.COM/SOCIEDAD/AUDIOS/E-SPORTS-ESPANAANDRES-CARABANTES/CSRCSRPOR/20140116CSRCSRSOC_22/AES/ (CONSULTADO 25/01/2014).

CPL, (2014): "THE CPL HERITAGE", EN HTTP://THECPL.COM/THE-CPL-HERITAGE/ (CONSULTADO 06/01/2014).

Del Palacio, Guillermo (2012): "De profesión, player" en El Mundo, 16 de junio de 2012.

HTTP://WWW.ELMUNDO.ES/ELMUNDO/2012/06/14/NAVEGANTE/1339685507.HTML (CONSULtADO: 06/01/2014).

Ewalt, David M. (2013): "The ESPN of Video games", en Forbes, 13 De noviembre 2013, EN HTTP://WWW.FORBES.COM/SITES/DAVIDEWALT/2013/11/13/THE-ESPN-OF-VIDEOGAMES/ (CONSULTADO: 06/01/2014).

Fernández, David (2013): "los esports en España. Charla con Sergi Mesonero, DIRECTOR DE LA LVP" EN HTTPS://WWW.YOUTUBE.COM/WATCH?V=H5I2SEC29GI (CONSULTADO: 07/01/2014).

Gómez, Salvador. (2007): "Videojuegos: el desafío de un nuevo medio a la COMUNICACIÓN SOCIAL", EN HISTORIA Y COMUNICACIÓN SOCIAL, No 12, 2007, PP. 7182. ISSN: $1137-0734$.

Gómez, Salvador; Navarro Nuria (2013): "Videojuegos e información. Una APROXIMACIÓN A LOS NEWSGAMES ESPAÑOLES COMO NUEVA ÓPTICA INFORMATIVA", EN ICONO 14, VOL. 11 (2), NO 2, 2013, PP. 31-51. ISSN: 1697-8293. DOI: RI14.V11I2.604.

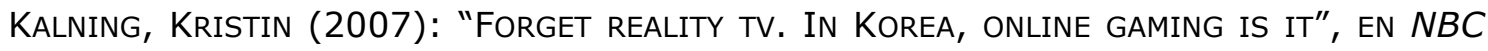
NEWS, 21 DE FEBRERO DE 2007, EN HTTP://WWW.NBCNEWS.COM/ID/17175353/ (CONSUltado: 05/01/2014).

LANG, DerRik J. (2013): "League of Legends champs Win in legendary Venue", en NBC NEWS, 7 DE OCTUBRE DE 2013, EN HTTP://WWW.NBCNEWS.COM/TECHNOLOGY/LEAGUELEGENDS-CHAMPS-WIN-LEGENDARY-VENUE-8C11339365

(CONSULTADO: 06/01/2014).

MARCANO, BEATRIZ (2012): "CARACTERÍSTICAS SOCIOLÓGICAS DE VIDEOJUGADORES ONLINE Y EL E-SPORT. El CASO dE CALL OF DUTY" EN PEDAGOGÍa SOCIAL. REVISTA INTERUNIVERSITARIA, No 19, 2012, PP. 113-124. ISSN: 1139-1723. DOI: 10.7179/PSRI.

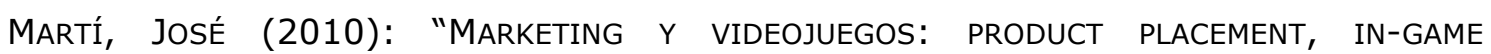
ADVERTISING Y ADVERGAMING", MADRID, ESIC. 
Martorell, Cristina; Rom, Josep A. (2011): "LA Cara oculta de los adVergames. La EXPLOTACIÓN CRÍTICA DE UN NUEVO RECURSO DE LA PUBLICIDAD", EN QUESTIONES PUBLICITARIAS, VOL. I, No 16, 2011, PP. 24-39. ISSN 1988-8732.

MEC (2008): "QUÉ ES EL STREAMING", EN HTTP://WWW.ITE.EDUCACION.ES/FORMACION/MATERIALES/107/CD/VIDEO/VIDEO0103.H TML

MONTERO, J. y RUEDA, J.C. (2001). INTRODUCCIÓN A LA HistoRia DE LA COMUNiCACión SOCIAL, MADRID, ARIEL.

MCU (2013): "ANUARIO DE ESTADÍSTICAS CULTURALES 2013 DEL MINISTERIO DE EDUCACIÓN, CULTURA Y DEPORTE", NOVIEMBRE DE 2013, EN HTTP://WWW.MCU.ES/ESTADISTICAS/DOCS/CAPITULOS_GRAFICOS/AEC2013/AEC_201 3.PDF (CONSULTADO: 08/01/2014).

mLG, (2013): "About Major league Gaming (MLG)", en HTTP://WWW.MAJORLEAGUEGAMING.COM/MLG/ABOUT (CONSULTADO 06/01/2014)

Nine Hour Films; Devolver Digital (2013): "Good Game Official Trailer", en HTTP://GOODGAMEMOVIE.COM/ (CONSULTADO: 06/01/2014).

Pascual, Juan Antonio (2013): "DreamHack Valencia 2013, el mayor festival de ESPORTS", EN COMPUTER HOY, 20 DE JULIO DE 2013, EN HTTP://COMPUTERHOY.COM/NOTICIAS/SOFTWARE/DREAMHACK-VALENCIA-2013-MAYORFESTIVAL-ESPORTS-5123 (CONSULTADO: 06/01/2014).

Ramírez, Mario (2008): "Organización de un eVento lan Party", Valladolid, UNIVERSIDAD DE VALLADOLID. DisPONIBLE EN HTTP://PFM.GOOGLECODE.COM/SVNHISTORY/R93/TRUNK/MEMORIA/PFM_LAN_PARTY.PDF (CONSULTADO: 06/01/2014).

Red Apple Entertainment (2006): "Gamer Revolution", Canada, 2006.

Riot, (2012): "League of legends' Growth Spells bad News for Teemo", en Riot GAMES, 15 DE OCTUBRE DE 2012, EN HTTP://WWW.RIOTGAMES.COM/ARTICLES/20121015/138/LEAGUE-LEGENDS-GROWTHSPELLS-BAD-NEWS-TEEMO (CONSULTADO: 06/01/2014).

TRASGO (2014): "S. RADU: «ESPAÑA ES UNA DE LAS GRANDES FUERZAS EUROPEAS EN ESPORTS»", EN TRASGO.NET, 4 DE ENERO DE 2014 EN HTTP://TRASGO.NET/NOTICIASESPORTS/GENERAL/ENTREVISTA-SEBASTIAN-RADU (CONSULTADO: 10/01/2014).

TWITCH, (2014): "All ABOUt TWITCH", EN HTTP://ES-ES.TWITCH.TV/P/ABOUT (CONSULTADO: $15 / 01 / 2014)$.

Wagner, Michael G. (2006): "On the Scientific Relevance of eSports", en Proceedings of INTERNATIONAL CONFERENCE ON INTERNET COMPUTING. PP. 437-442. DisPonible EN HTTP://WW1.UCMSS.COM/BOOKS/LFS/CSREA2006/ICM4205.PDF (CONSULTADO: 29/01/2014). 
[Recibido: 30 de enero de 2014. Aceptado con cambios: 1 de octubre de 2014 . Aceptación definitiva: 12 de diciembre de 2014]. 